\title{
LOCALIZATION OF A CAR BASED ON MULTI-SENSOR FUSION
}

\author{
H. Kim ${ }^{1}$, I. Lee ${ }^{1, *}$ \\ ${ }^{1}$ Dept. of Geoinformatics, The University of Seoul, 163 Seoulsiripdaero, Dongdaemun-gu, Seoul, Korea - (zzimss, iplee)@uos.ac.kr
}

Commission I, WG I/6

KEY WORDS: Navigation, Positioning, Kalman Filter, Sensor Fusion

\begin{abstract}
:
The vehicle localization is an essential component for stable autonomous car operation. There are many algorithms for the vehicle localization. However, it still needs much improvement in terms of its accuracy and cost. In this paper, sensor fusion based localization algorithm is used for solving this problem. Our sensor system is composed of in-vehicle sensors, GPS and vision sensors. The localization algorithm is based on extended Kalman filter and it has time update step and measurement update step. In the time update step, in-vehicle sensors are used such as yaw-rate and speed sensor. And GPS and vision sensor information are used to update the vehicle position in the measurement update step. We use visual odometry library to process vision sensor data and generate the moving distance and direction of the car. Especially, when performing visual odometry we use georeferenced image database to reduce the error accumulation. Through the experiments, the proposed localization algorithm is verified and evaluated. The RMS errors of the estimated result from the proposed algorithm are about $4.3 \mathrm{~m}$. This result shows about $40 \%$ improvement in accuracy even in comparison with the result from the GPS only method. It shows the possibility to use proposed localization algorithm. However, it is still necessary to improve the accuracy for applying this algorithm to the autonomous car. Therefore, we plan to use multiple cameras (rear cameras or AVM cameras) and more information such as high-definition map or V2X communication. And the filter and error modelling also need to be changed for the better results.
\end{abstract}

\section{INTRODUCTION}

The vehicle localization is an important component for stable autonomous car operation. The most efficient and accurate way for positioning is Global Navigation Satellite System (GNSS) such as Global Positioning System (GPS). GPS is the most common method to get the position for many purpose. However, it may not always provide accurate result because of the week or wrong signal due to the urban environments. Therefore, many researches are focused on supplementing the GPS accuracy. The most common method is to use additional sensors. Many automobile production companies already developed autonomous car positioning algorithm and they are now focusing on stability improvement. They developed a high precision localization system using high-cost GPS and IMU sensors for high performances (Franke et al., 2013). And some companies such as Google also use Lidar sensors and radar sensors together for precise localization and perception. However, the cost of sensors that they used are very high and it is not suitable for commercialized cars.

Therefore, there are many attempts to replace high-priced sensors with inexpensive sensors using a sensor fusion algorithm. One of the typical attempts is to combine GPS and in-vehicle sensors (Jo et al., 2012). In commercial cars, there are some on-board sensors such as speed and yaw-rate sensors. And these sensors can replace the role of expensive IMU sensors. But still there is accuracy problem. Because this method has a problem with the error accumulation.

Some other papers use vision sensors. The price of vision sensor is low but the information from them are helpful for estimating the vehicle position. The lane marking detection for localization is one of the research that can improve the accuracy of the localization (Lu et al., 2014). Visual odometry approach can compute the relative poses in the sequential images in real time (Nister et al., 2004). Nath et al. (2012) developed an algorithm to estimate 3D position of moving platform using least squares estimation strategy. The combination of GPS and video camera was also studied (Soloview and Venable, 2010).

Map-based localization algorithm also can be a one of the method to reduce errors. For autonomous vehicle, many company made an accurate digital map called high definition map (Karlsson and Gustafsson, 2017). It contains geographic information such as digital elevation model (DEM) and landmarks information such as traffic lights or speed-limit signs. There are many studies using digital map and geographical information for localization. DEM information is used for estimating the positions and attitudes of a vehicle (Larnaout et al., 2012) and 3D point cloud database is employed for vehicle localization (Yoneda et al., 2014). In addition, traffic sign image database or road lane information in the digital map is also used for vehicle localization (Vivaqua et al., 2018). Furthermore, 3D building map-based localization was studied (Shunsuke et al., 2015). It uses 3D building map based GNSS Positioning, on-board sensors for localization. And monocular camera is used to achieve lane level accuracy.

This paper presents a car localization method using multiple costeffective sensors. For realizing our localization method to commercialized car, we adopted the sensors that are cheap and easy to get and install such as in-vehicle sensors, a low-cost GPS and cameras. And we designed the localization framework based on sensor fusion method. The extended Kalman filter is used to integrate the information from multiple sensor measurements. To check the feasibility of proposed localization method, we built the sensory data acquisition system and mounted on the vehicle. During the driving on real road, the system gets the sensor data and use it as inputs for proposed localization algorithm. As a result of the proposed algorithm, there is an accuracy improvement compared it with other localization methods.

\footnotetext{
* Corresponding author
} 


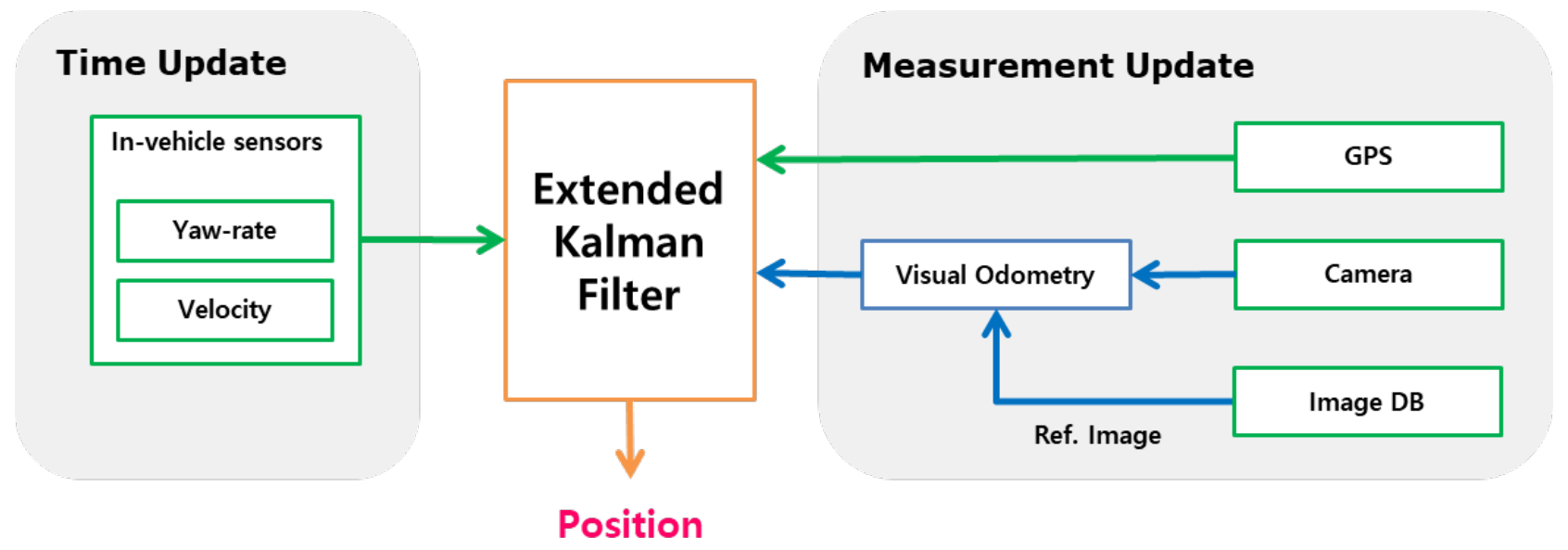

Figure 1. Overall framework of the localization algorithm.

The rest of this paper is organized as follows. Section II describes the flow of the localization framework. Section III presents data data acquisition and experimental results for the proposed algorithm and the conclusions are followed in final section.

\section{LOCALIZATION FRAMEWORK}

Figure 1 represents the overall framework of the positioning system using sensor fusion algorithm. The extended Kalman filter is used to integrate the sensors information. The process of this system is composed of two parts. One part is time update and the other part is measurement update. At the time update step, vehicle state is predicted which is based on the bicycle model. And at the measurement update step, the GPS measurements and visual odometry results are used for updating the vehicle position estimation. The time update step and measurement update step are performed repeatedly. Through the GPS and visual information, it can estimate the horizontal and vertical position. However, the horizontal position information is more important in the autonomous vehicle. And to simplify the estimation model, we only consider the horizontal position in this framework.

\subsection{Time update}

In the time update step, the system updates the vehicle state using bicycle model with in-vehicle sensors inputs (speed and yaw rate) The equation (1) is shown how to update the vehicle state. This model contains three states: 2D vehicle coordinates (X, Y) and vehicle heading $(\theta)$.

$\hat{x}_{k \mid k-1}=\left[\begin{array}{c}X_{k \mid k-1} \\ Y_{k \mid k-1} \\ \theta_{k \mid k-1}\end{array}\right]=\left[\begin{array}{c}X_{k-1}+\Delta T \cdot V \cdot \cos \left(\theta_{k-1}+\Delta T \cdot \omega\right) \\ Y_{k-1}+\Delta T \cdot V \cdot \sin \left(\theta_{k-1}+\Delta T \cdot \omega\right) \\ \theta_{k-1}+\Delta T \cdot \omega\end{array}\right]$

where $\quad x=$ state vector

$X, \mathrm{Y}=$ vehicle coordinates

$\theta=$ vehicle heading

$V=$ speed

$\Delta T=$ time elapsed

$\omega=$ yaw-rate

Through this step, the position and driving direction of the car can be estimated continuously with the passage of time. And the system can determine the current position and driving direction even if there is no information obtained from the outside of vehicle such as GPS.

\subsection{Measurement Update}

In this paper, there are two types of measurement update. One is a GPS measurement and other is a visual odometry measurement. The system monitors which sensor is newly observed. And when a new sensor data is measured, the measurement value is reflected the system and it update the vehicle state.

2.2.1 GPS Update: In the GPS update step, the system obtains the positioning information from GPS receiver. Whenever the new GPS signal is received, the car position is updated using this information. The measurement vector can be expressed as equation (2).

$$
\mathrm{z}_{\mathrm{k}}=\left[X_{G P S} Y_{G P S}\right]^{T}
$$

where $X_{G P S}$ and $Y_{G P S}$ represent the global position from the GPS receiver.

In this system, we only consider the information about the horizontal position. Therefore, we just use the $\mathrm{X}$ and $\mathrm{Y}$ values and ignore the $\mathrm{Z}$ value from GPS signal.

2.2.2 Visual Odometry Update: In this step, the system updates the position using visual odometry result. Through the visual odometry, the system can estimate movement of the car. We use LIBVISO2 which is open source library for visual odometry (Geiger et al., 2011). This library works well for both circumstance mono and stereo front-view camera. The measurement vector can be expressed as equation (3).

$$
\mathrm{z}_{\mathrm{k}}=\left[\begin{array}{lll}
X_{V O} & Y_{V O} & \theta_{V O}
\end{array}\right]^{T}
$$

where $X_{V O}$ and $Y_{V O}$ represent the global position from the visual odometry result and $\theta_{V O}$ represent the vehicle driving direction also from the visual odometry result.

Furthermore, the system uses image database estimation for estimating the car position more accurately. Dead reckoning in time update step, GPS and visual odometry in measurement update step are a good solution for estimating a car position. However, it is not stable solution. Because dead reckoning sensors and visual sensors are noisy, and they have integration errors. And GPS outage occur frequently in urban environments. Consequently, the localization accuracy is not enough for selfdriving car. To overcome these problems, this system uses georeferenced image-based localization algorithm. Before driving, 
we acquired images and corresponding geometric information such as position and attitude. And the system storing these georeferenced image data. The system uses frontal image sequence and information of geo-referenced images together to get the refined results. In this part, whenever corresponding georeferenced image is coming up we load this image information and combine it with frontal image. This information works the similar role as a map. The concept of localization using georeferenced image is shown in figure 2 . This Through this process, the system can reduce the accumulated errors from vision sensors and estimate the car position more precisely.

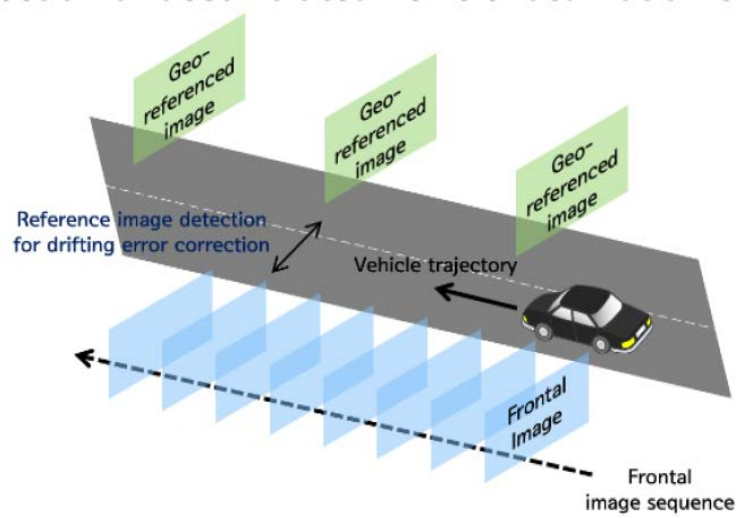

Figure 2. Concept of the localization using image database

\section{EXPERIMENT}

For testing the proposed algorithm, we build a data acquisition system. Figure 3 is shown some part of our system. Our sensor system is composed of in-vehicle sensors, a low-cost GPS receiver and two front-view cameras. Furthermore, the reference data measurement equipment is also mounted on the car for accuracy evaluation. We estimate the vehicle position using proposed localization framework and compare it with the true position which is determined by reference equipment.

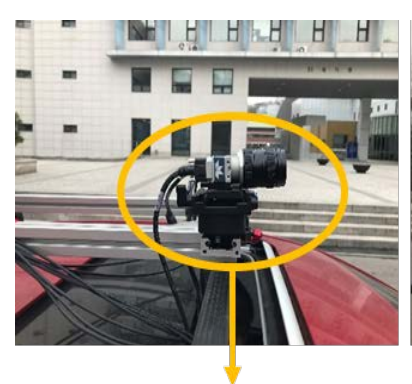

Front cameras

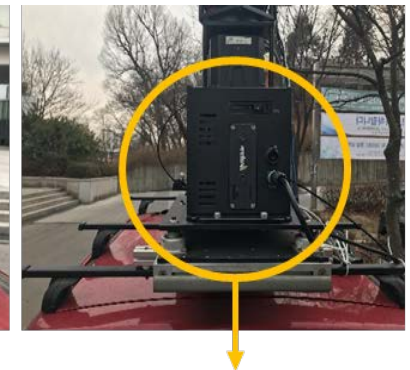

Reference Equipement
Figure 3. The data acquisition system

\subsection{Experimental System Configuration}

We set a test vehicle equipped with data acquisition system. This system can acquire the in-vehicle sensors data such as yaw rate and vehicle speed using Controller Area Network (CAN) bus. We use the Kvaser USBcan Pro interface for logging the in-vehicle sensors data. The data from the in-vehicle sensors are used to estimate the car motion. A low-cost GPS receiver is also used for determining the position of the car. We use a smartphone (Samsung Galaxy S6) for GPS receiver and save the signal log to the laptop. In addition, we also use the two front-view cameras for obtaining driving images. We adopt visual odometry algorithm called LIBVISO2 to estimate the relative movement and direction of the car. For this visual odometry process, we installed two front view cameras (Genie Nano) and suitable lens (Pomeas) on the roof of the car. The specifications of the image sensors are listed in Table 1 . And for the evaluation, we also installed Applanix POSLV 210 which is a high-accurate data acquisition device.

\begin{tabular}{ccc}
\hline Parameter & Value & Unit \\
\hline Focal length & 12 & $\mathrm{~mm}$ \\
Resolution & $2592 \times 2048$ & pixels \\
Pixel size & $4.8 \times 4.8$ & $\mu \mathrm{m}$ \\
\hline
\end{tabular}

Table 1. The image sensor specification

\subsection{Experimental Results}

The proposed localization algorithm is evaluated through the experiment. To analyse the proposed localization algorithm, we conducted experiment with acquired data. The driving data was acquired near the University of Seoul, Korea. The trajectory is shown in figure 4 . It took around 407 seconds and the driving distance was $1.4 \mathrm{~km}$. And the GPS data acquisition rate was $1 \mathrm{~Hz}$ and images from front cameras were captured in every 2 meters driving distance. In this experiment, it is assumed that the georeferenced images are existed in every 40 meters. The highaccurate driving data acquisition system was used to evaluate the accuracy of proposed method and we regarded it as a reference value. The accuracy was evaluated based on the position difference between each localization method and the reference driving data. The localization estimation results are shown in figure 5 . The blue line indicates the trajectory from reference device. The red line and yellow line are the trajectories determined from the in-vehicle sensor information and the GPS signal respectively. And the purple line indicates the trajectory from proposed localization method.

Figure 5 shows the distance differences comparing to the reference data. The proposed sensor fusion method shows more stable and accurate result then other sensors. And the table 2 indicates the error statistics comparing with the reference data. The RMS of the dead reckoning only method and the RMS of the GPS only method is about 35.1 meters and 7.2 meters respectively. But the RMS of the proposed sensor fusion method is only 4.3 meters. The result shows that the estimation performance of proposed method is improved about 41\% compared with GPS only method.

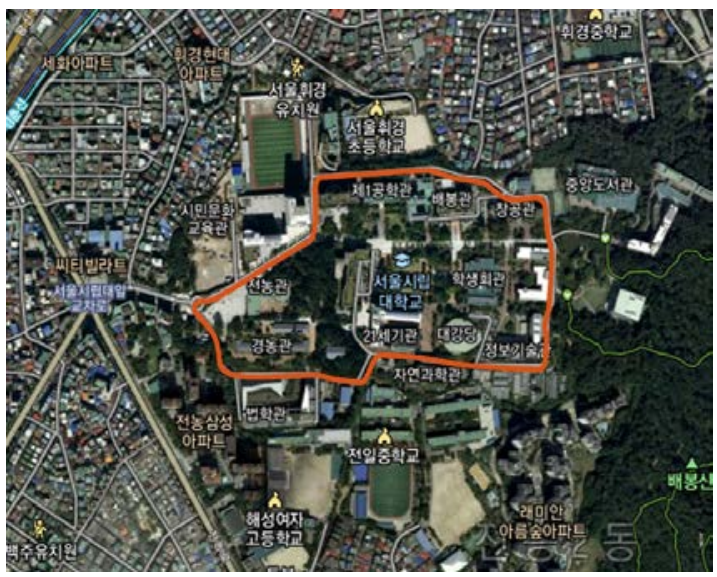

Figure 4. The test site and trajectory (red line) 


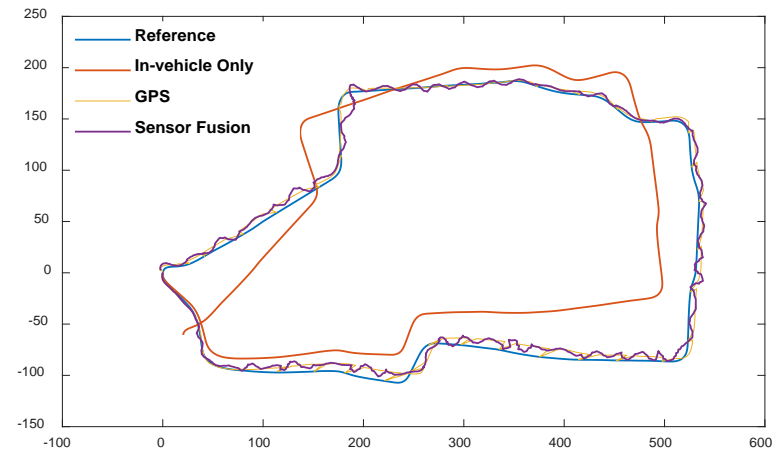

Figure 5. Trajectory comparison

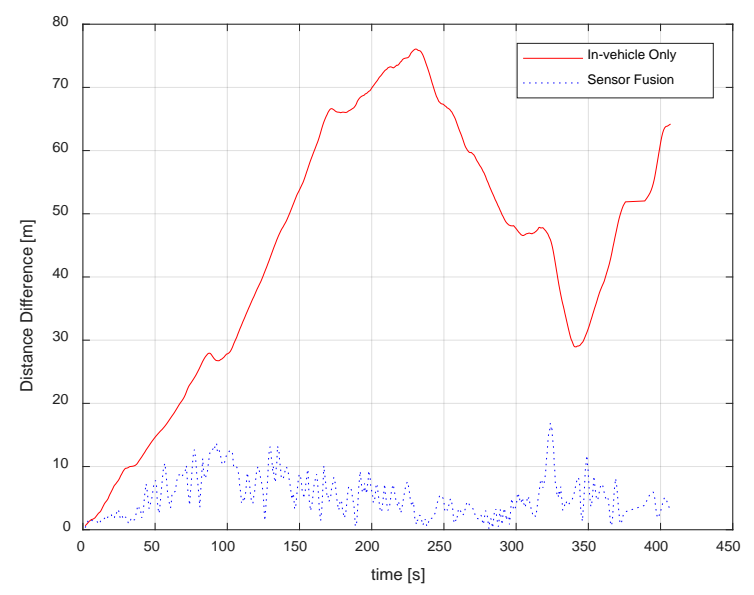

Figure 6. Comparison of position difference

\begin{tabular}{cccccc}
\hline Unit $[\mathrm{m}]$ & Mean & STD & RMS & Min & Max \\
\hline CAN & 4.03 & 34.8 & 35.1 & -61.8 & 60.9 \\
GPS & -3.1 & 6.6 & 7.2 & -19.9 & 14.4 \\
Sensor Fusion & -2.0 & 3.8 & 4.3 & -15.8 & 7.9 \\
\hline
\end{tabular}

Table 2. Errors of position estimation

\section{CONCLUSION}

This paper proposed a localization algorithm based on sensor fusion algorithm. This algorithm integrates speed sensor, yawrate sensor, GPS and vision sensors using extended Kalman filter method. And it also uses image database in visual odometry step for reduce the error accumulation.

The proposed localization algorithm can operate with low-cost sensors and it can reduce errors using sensor fusion. However, it is still necessary to improve the localization accuracy for the autonomous car. In order to reduce the positioning errors, we plan to integrate other sensors such as AVM cameras, precise digital map and V2X devices. The errors will be reduced when the system integrates additional sensors. The filter and error modelling will need to be changed for additional sensor information.

\section{ACKNOWLEDGEMENTS}

This research was supported by Basic Research Program through the National Research Foundation of Korea (NRF) funded by the
Ministry of Science, ICT \& Future Planning (NRF2017R1A2B4012908).

\section{REFERENCES}

Franke, U., Pfeiffer, D., Rabe, C., Knoeppel, C., Enzweiler, M., Stein, F., Herrtwich, R.G., 2013. Making bertha see. In: Proceedings of the IEEE International Conference on Computer Vision Workshops, pp. 214-221.

Geiger, A., Ziegler, J., Stiller, C., 2011. StereoScan: Dense 3d reconstruction in real-time. In: 2011 IEEE Intelligent Vehicles Symposium (IV), pp. 963-968.

Jo, K., Chu, K., Sunwoo, M., 2012. Interacting Multiple Model Filter-Based Sensor Fusion of GPS With In-Vehicle Sensors for Real-Time Vehicle Positioning. IEEE Intelligent Transportation Systems, 13(1), pp. 329-343.

Karlsson, R., Gustafsson, F., 2017. The Future of Automotive Localization Algorithms: Available, reliable, and scalable localization: Anywhere and anytime. IEEE Signal Process, 34(2), pp. 60-69.

Larnaout, D., Bourgeois, S., Gay-Bellile, V., Dhome, M., 2012. Towards Bundle Adjustment with GIS Constraints for Online Geo-Localization of a Vehicle in Urban Center. In: 2012 Second International Conference on 3D Imaging, Modeling, Processing, Visualization \& Transmission. IEEE, pp. 348-355.

Lu, W., Seignez, E., Rodriguez, F.S.A., Reynaud, R., 2014. Lane marking based vehicle localization using particle filter and multikernel estimation. In: 2014 13th International Conference on Control Automation Robotics \& Vision (ICARCV). IEEE, pp. 601-606.

Nath, N., Dawson, D.M., Tatlicioglu, E., 2012. Euclidean Position Estimation of Static Features Using a Moving Uncalibrated Camera. Control Systems Technology IEEE Transactions on, 20(2), pp. 480-485.

Nister, D., Naroditsky, O., Bergen, J., 2004. Visual odometry. In: Proceedings of the 2004 IEEE Computer Society Conference on Computer Vision and Pattern Recognition, pp. 652-659.

Soloviev, A., Venable, D., 2010. Integration of GPS and vision measurements for navigation in GPS challenged environments. In: Position Location and Navigation Symposium (PLANS), 2010 IEEE/ION, pp. 826-833.

Shunsuke, K., Yanlei, G., Hsu, L.-T., 2015. GNSS/INS/On-board Camera Integration for Vehicle Self-Localization in Urban Canyon. In: 2015 IEEE 18th International Conference on Intelligent Transportation Systems. IEEE, pp. 2533-2538.

Vivacqua, R.P.D., Bertozzi, M., Cerri, P., Martins, F.N., Vassallo, R.F., 2018. Self-Localization Based on Visual Lane Marking Maps: An Accurate Low-Cost Approach for Autonomous Driving. IEEE Intelligent Transportation System, 19(2), pp. 582597.

Yoneda, K., Tehrani, H., Ogawa, T., Hukuyama, N., Mita, S., 2014. Lidar scan feature for localization with highly precise 3-D map. In: 2014 IEEE Intelligent Vehicles Symposium Proceedings. IEEE, pp. 1345-1350. 